\title{
Application of Digital Volume Correlation to X-ray Computed Tomography Images of Shale
}

\author{
Tae Wook Kim, Wonjin Yun, and Anthony R. Kovscek* \\ Energy Resources Engineering \\ Stanford University, Stanford CA 94305 USA \\ *email: kovscek@stanford.edu
}

\section{Supporting Information}

\section{Experimental conditions}

The experimental conditions of temperature and stress for samples GRC5, GRH7, GRS4, and GRS6 are found in Figure S1. Samples GRS4 and GRS6 were used to measure Young's modulus prior to and post maturation.

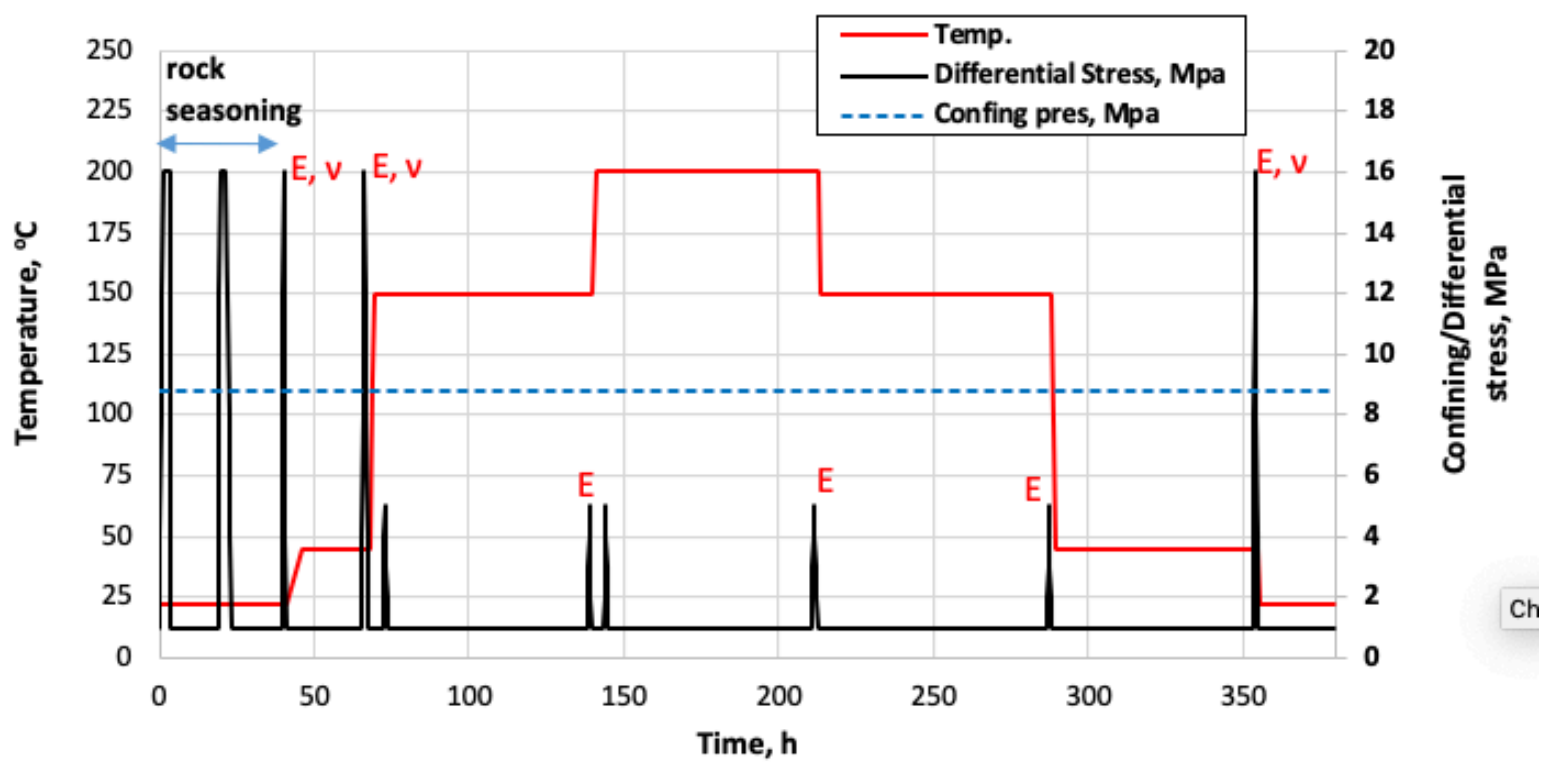

(a) 


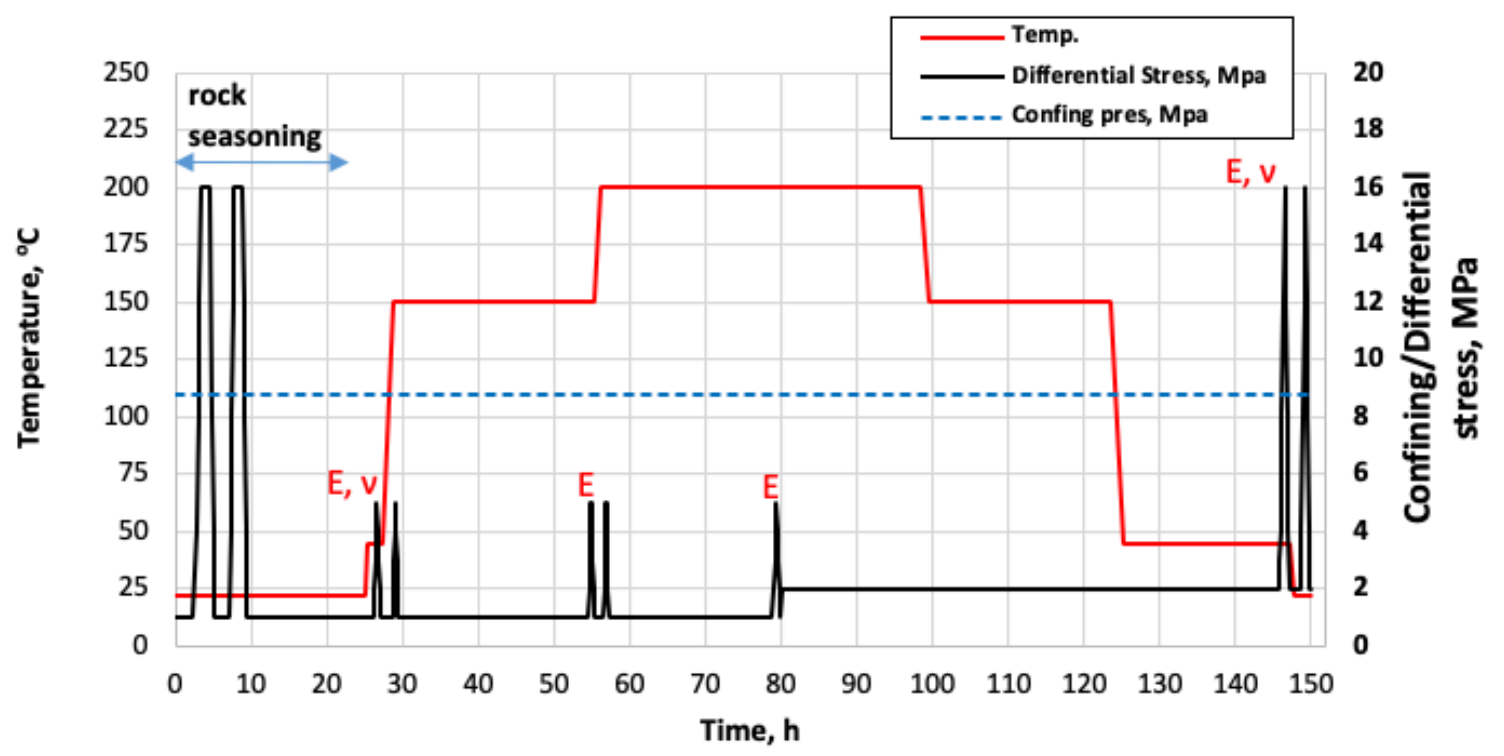

(b) 


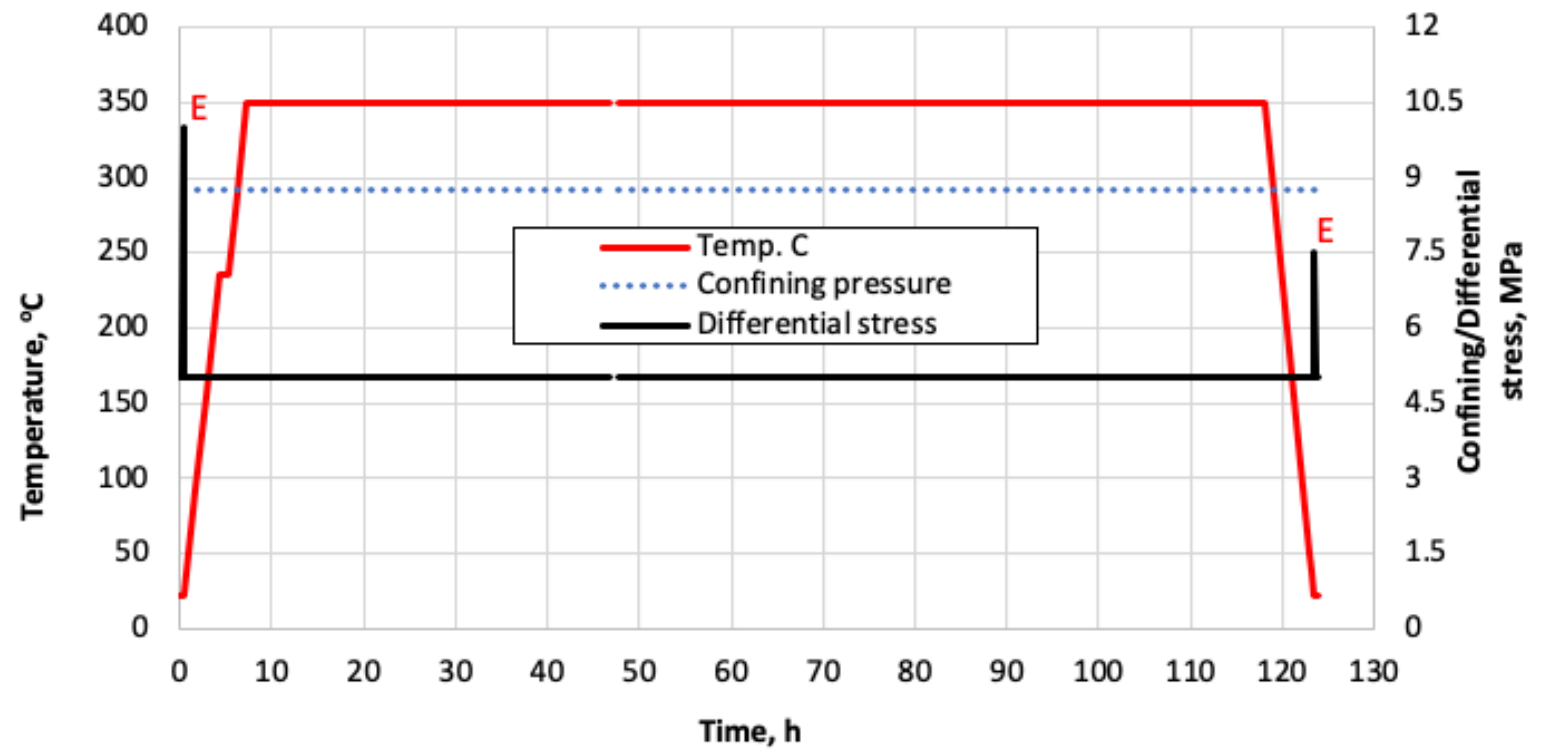

(c)

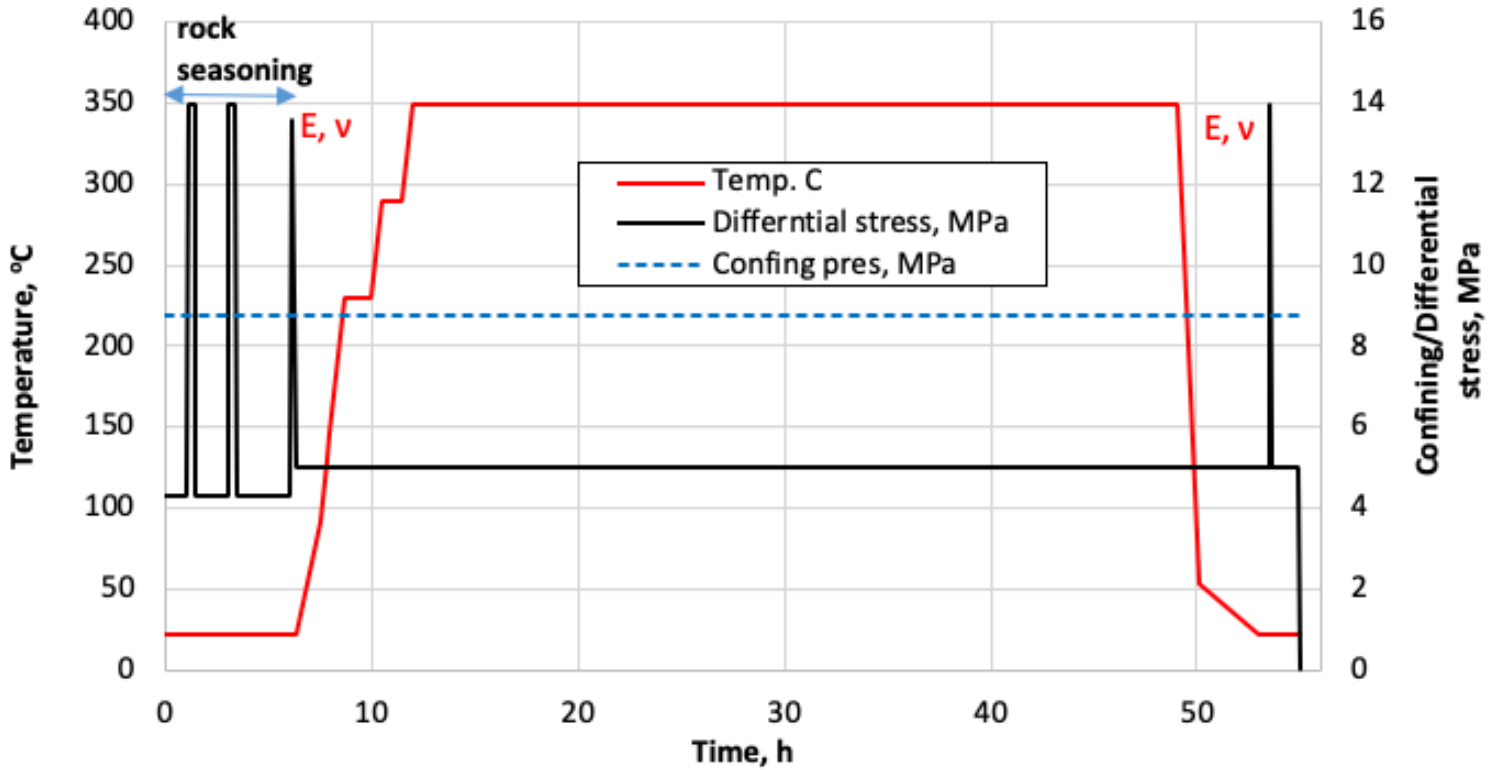

(d)

Figure S1 Experimental temperature and stress conditions for (a) GRC5, (b) GRH7, (c) GRS4, and (d) GRS6. (E: Young's modulus measurement, v: Poisson's ratio measurement). 


\section{Semi-quantitative XRD analysis}

Table S1 shows the composition of minerals from semi-quantitative XRD used for the ternary plot in Fig. 5(a). The carbonate component is the sum of calcite and dolomite. The clay component is the sum of illite and other clays.

Table S1 Mineral composition from semi-quantitative XRD analysis.

\begin{tabular}{|c|c|c|c|c|c|c|c|}
\hline \multirow{2}{*}{ Sample } & \multicolumn{9}{|c|}{ Composition, wt \% } \\
\cline { 2 - 9 } & Calcite & Dolomite & Quartz & Pyrite & Feldspar & Illite & $\begin{array}{c}\text { Other } \\
\text { Clays }\end{array}$ \\
\hline GRS1 & 9.3 & 65 & 17.6 & - & 4.5 & 3.6 & - \\
\hline GRS3 & 5.8 & 61 & 9.7 & 1.1 & 21.7 & 0.6 & 0.1 \\
\hline GRS4 & 11.4 & 56 & 17.7 & 0.2 & 10.3 & 4.3 & 0.1 \\
\hline GRS6 & 10.7 & 58 & 5.4 & 0.1 & 8.8 & 17 & - \\
\hline GRH1 & 6.8 & 63 & 13 & 0.01 & 8.2 & 9 & - \\
\hline GRH4 & 12 & 49 & 20 & 0.5 & 13.8 & 0.2 & 4.5 \\
\hline GRH7 & 10.7 & 69.9 & 14 & - & 4.3 & 1.1 & - \\
\hline GRC5 & 13 & 40 & 7 & 0.1 & 39.6 & 0.3 & - \\
\hline
\end{tabular}

\section{Poisson's ratio data}

Table S2 shows Poisson's ratio from axial and radial strain. The applied effective differential stresses were identical except sample GRH7.

Table S2 Poisson's ratio.

\begin{tabular}{|c|c|c|c|c|c|c|}
\hline $\begin{array}{c}\text { Sample } \\
\text { (size) }\end{array}$ & Status & $\begin{array}{c}\text { Temp. } \\
{ }^{\circ} \mathrm{C}\end{array}$ & Axial strain & $\begin{array}{c}\text { Effective } \\
\text { differential } \\
\text { stress }\end{array}$ & Radial strain & Poisson ratio \\
\hline \multirow{2}{*}{ GRS6 } & Prior-maturation & \multirow{2}{*}{22} & -0.00202 & 9.3 & 0.00026 & $0.16 \pm 0.016$ \\
\hline & Post-maturation & & -0.00431 & 8.7 & 0.0016 & $0.38 \pm 0.03$ \\
\hline \multirow{2}{*}{ GRH1 } & Prior-maturation & \multirow[t]{2}{*}{45} & -0.00135 & 7.1 & 0.00016 & $0.12 \pm 0.018$ \\
\hline & Post-maturation & & -0.00172 & 7.0 & 0.000754 & $0.44 \pm 0.04$ \\
\hline \multirow{2}{*}{ GRH7 } & Prior-pyrolysis & \multirow{2}{*}{45} & -0.00162 & 4 & 0.000125 & $0.08 \pm 0.01$ \\
\hline & Post-pyrolysis & & -0.00402 & 14 & 0.000426 & $0.11 \pm 0.01$ \\
\hline \multirow{2}{*}{ GRC5 } & Prior-pyrolysis & \multirow{2}{*}{45} & -0.00198 & 15 & 0.000168 & $0.09 \pm 0.01$ \\
\hline & Post-pyrolysis & & -0.00277 & 15 & 0.000237 & $0.09 \pm 0.01$ \\
\hline \multirow{2}{*}{ GRS1 } & Prior-pyrolysis & \multirow{2}{*}{45} & -0.00365 & 14 & 0.000152 & $0.04 \pm 0.005$ \\
\hline & Post-pyrolysis & & -0.00360 & 14 & 0.0001778 & $0.05 \pm 0.006$ \\
\hline
\end{tabular}




\section{Micro-CT of GRH7}

Micro-CT (Zeiss Xradia 520 Versa X-ray CT) imaging provides images of fractures in GRH7. The X-ray source voltage and power settings were $140 \mathrm{kV}$ and $10 \mathrm{~W}$, respectively. The voxel resolution is $30 \times 30 \times 30 \mu \mathrm{m}$ to capture the whole sample. After reconstructing the tomographic dataset, Avizo 9.0.1 software was used for further processing and analysis. Figure S2 shows fractures in $\mathrm{XY}$ and $\mathrm{YZ}$ planes as well as 3D images. Note the fractures in the longitudinal direction.

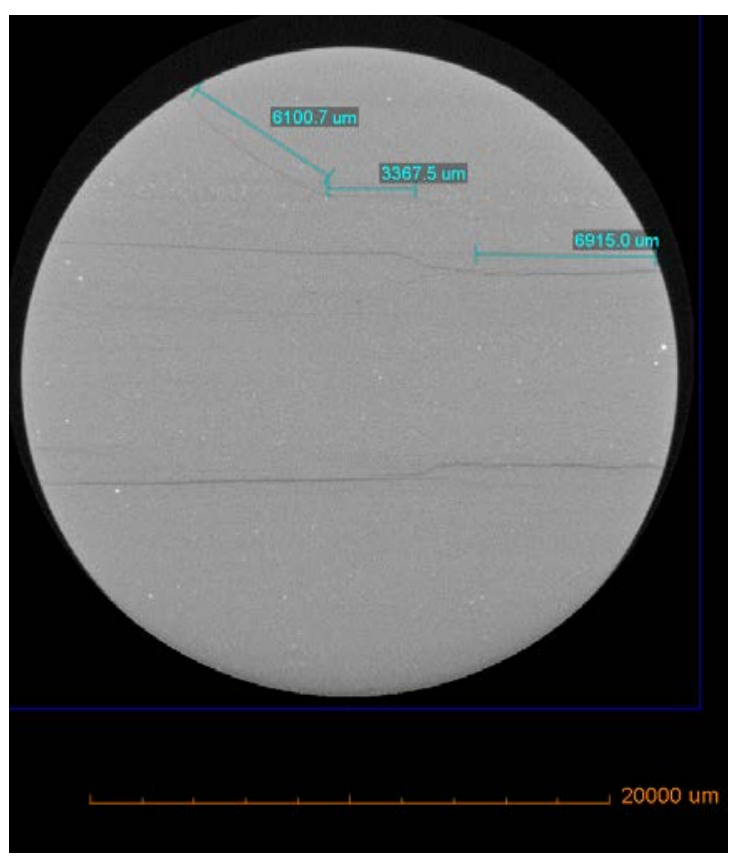

(a)

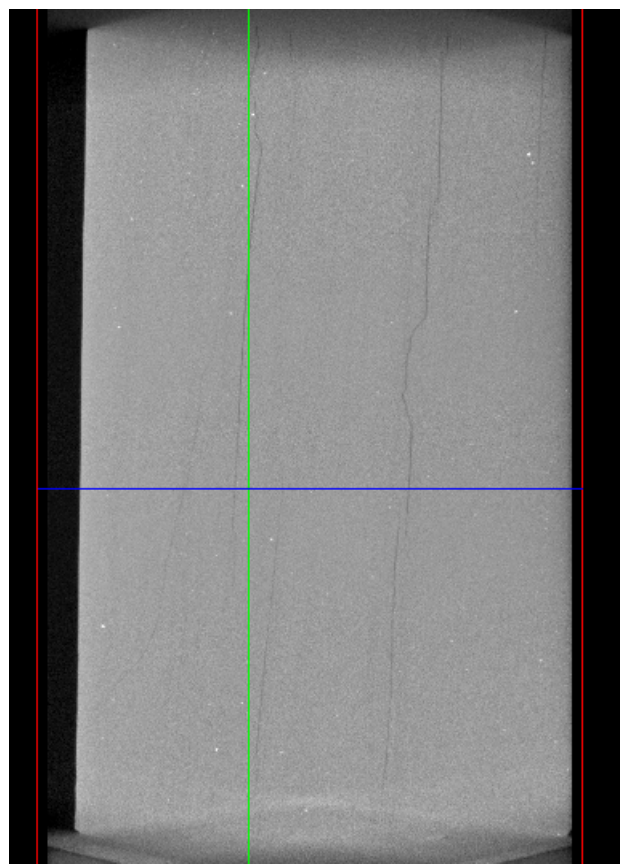

(b)

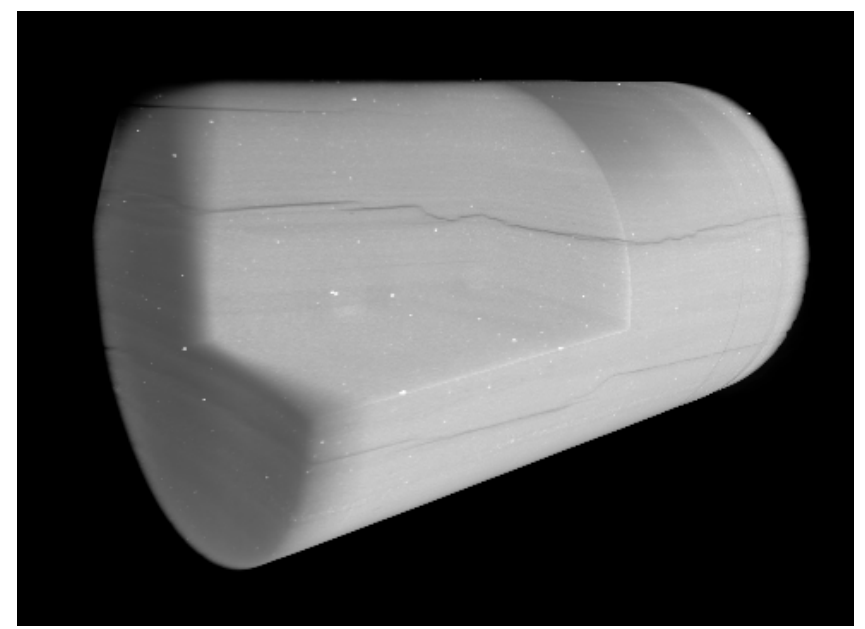

(c)

Figure S2 Fracture images of GRH7 for (a) XY plane, (b) YZ plane, and (c) 3D. 